\section{Discrete arcs of increased fundus autofluorescence in retinal dystrophies and functional correlate on microperimetry}

\begin{abstract}
Purpose To describe the occurrence of discrete arcs of increased fundus autofluorescence (FAF) associated with various retinal dystrophies and to assess their functional significance by fundus-controlled microperimetry.

Methods Seven patients, three with pigmented paravenous retinochoroidal atrophy (PPRCA), one with sector retinitis pigmentosa (RP), one with typical RP, and two with macular dystrophy were assessed by retinal imaging including FAF imaging. Serial images were obtained within a review period of 6 and 10 years in a patient with PPRCA and macular dystrophy, respectively. Funduscontrolled microperimetry was performed in eight eyes of five patients to determine light increment sensitivity.
\end{abstract}

Results A discrete arched line of increased FAF was observed without obvious correlate on fundus biomicroscopy. The orientation of this line differed from ring shape in RP and macular dystrophy, a semi-circle structure in sector RP to crescent shape with tiplike extensions towards branching retinal veins in PPRCA. Longitudinal investigation revealed slight migration of the arc in PPRCA and peripheral extension of the ring diameter in macular dystrophy. Microperimetry revealed that the arc of increased FAF sharply delineated areas of severely impaired retinal sensitivity.

Conclusions The findings indicate that arcs of increased FAF in PPRCA and other retinal dystrophies demarcate areas of impaired retinal function and may migrate over time. FAF imaging may therefore reveal the exact

M Fleckenstein, $\mathrm{P}$ Charbel Issa, HA Fuchs, RP Finger, H-M Helb, HPN Scholl and FG Holz

extent of neurosensory dysfunction that may exceed the dimensions anticipated by conventional examinations.

Eye (2009) 23, 567-575; doi:10.1038/eye.2008.59; published online 14 March 2008

Keywords: fundus autofluorescence; microperimetry; retinal dystrophies

\section{Introduction}

In vivo fundus autofluorescence (FAF) imaging allows visualisation of lipofuscin on the level of the retinal pigment epithelium (RPE) cell monolayer. ${ }^{1-5}$ As shown by Delori and coworkers, the FAF signal mainly originates from dominant fluorophores in cytoplasmic lipofuscin granules of the RPE. ${ }^{3}$ Lipofuscin accumulates with age in normal subjects and may increase or decrease abnormally due to retinal disease. ${ }^{6}$ FAF imaging provides information that is invisible to conventional fundus photography or fluorescein angiography. Both, its impact on more refined phenotyping and its potential as prognostic marker have been demonstrated previously in eyes with various manifestations of age-related macular degeneration. ${ }^{7-11}$

In retinal dystrophies, various topographic changes in FAF have been described.,12,13 The extent and pattern of abnormal FAF may show distinctive distributions in different forms of retinal dystrophies. Recently, in patients with retinitis pigmentosa (RP), ${ }^{14-19}$ Leber's congenital amaurosis (LCA), ${ }^{19,20}$ bull's eye maculopathy, ${ }^{21} \mathrm{X}$-linked retinoschisis (XLRS), ${ }^{19,22}$ best macular dystrophy, ${ }^{23}$ cone
Department of Ophthalmology, University

of Bonn, Bonn, Germany

Correspondence: FG Holz, Department of

Ophthalmology, University of Bonn Ernst-Abbe-Str. 2, Bonn 53127,

Germany

Tel: + 4922828715647 ; Fax: + 4922828715603

E-mail: Frank.Holz@

ukb.uni-bonn.de

Received: 2 October 2007 Accepted in revised form: 4 February 2008 Published online: 14 March 2008 
dystrophy, and cone-rod dystrophy, ${ }^{19,24-28}$ a parafoveal ring of increased FAF has been noted that may not be visible on biomicroscopy. In RP, constriction of this ring has been noted in some individuals over time. ${ }^{16,19}$ Recently, enlargement of the ring in two patients with cone-rod dystrophy has been reported. ${ }^{28}$ Functional assessment in patients with RP and good visual acuity revealed that the ring may encircle areas of preserved photopic function. ${ }^{14-19,28}$ However, the functional significance of this phenomenon detected by FAF imaging is incompletely understood. Furthermore, it is not known whether similar abnormal FAF alterations at the level of the RPE cell monolayer in various retinal disease share common underlying pathophysiological mechanisms. Here we investigated abnormal FAF findings in a spectrum of retinal dystrophies and explored the functional significance of abnormal FAF signals topographically by using fundus-controlled microperimetry. Moreover, we investigated in a subset of patients if the arc of increased FAF exhibits topographical variation over time.

\section{Materials and methods}

\section{Patients}

Seven patients were examined: three patients with pigmented paravenous retinochoroidal atrophy (PPRCA; patients 1-3), one patient with bilateral sector RP (patient 4), one patient with bilateral typical autosomal dominant RP (patient 5), and two patients with macular dystrophy (patients 6 and 7) (Table 1). The patients were investigated by routine ophthalmic examinations including best-corrected visual acuity, biomicroscopy, digital fundus photography, FAF imaging, visual field examinations (automated and static perimetry and manual kinetic perimetry), and full-field

electroretinography (ERG) according to the International Society for Clinical Electrophysiology in Vision standard $^{29}$ (Table 2).

Serial FAF imaging was performed in patient 1 in the years 1999, 2002, and 2005; in patient 6, FAF images were obtained in 1997 and 2007. In eight eyes of five patients, fundus-controlled microperimetry was performed.

The case history of patient 1 is described in detail. The study was in adherence to the tenets of the Declaration of Helsinki. Informed consent was obtained from each patient.

\section{FAF imaging}

FAF imaging was performed using a confocal scanning laser ophthalmoscope (Heidelberg Retina Angiograph, HRA classic and HRA II, Heidelberg Engineering,

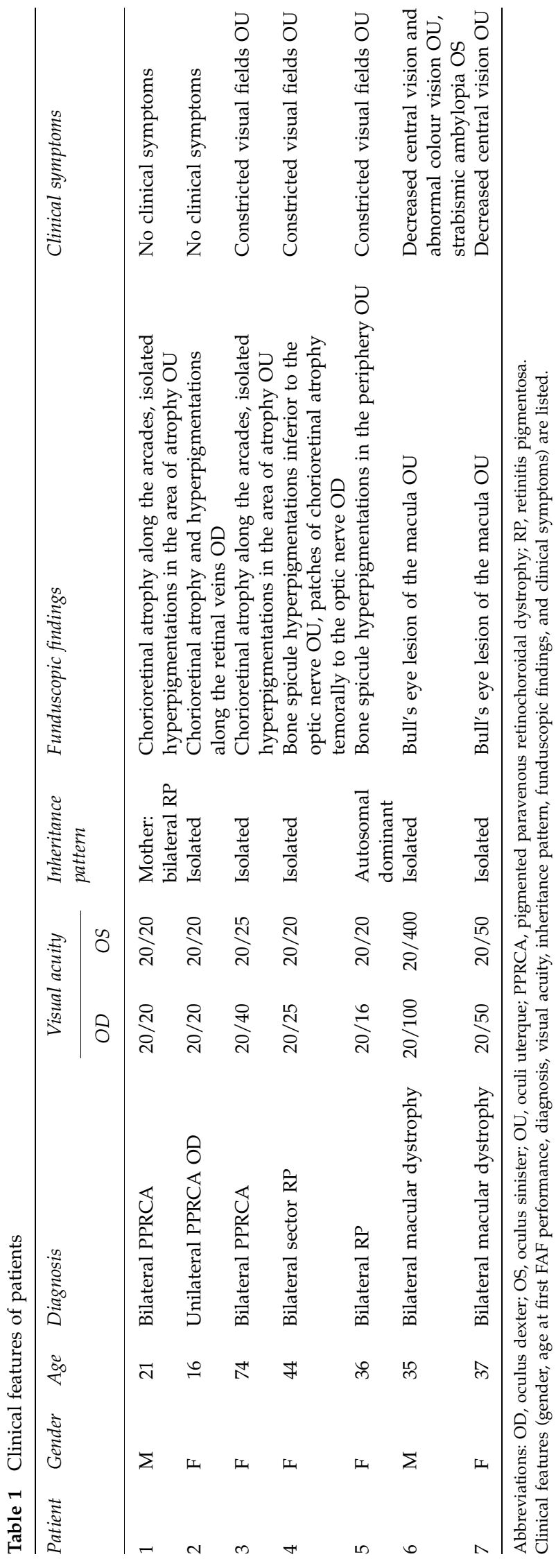


Table 2 Description of full-field ERGs

\begin{tabular}{|c|c|c|c|c|}
\hline \multirow[t]{3}{*}{ Patient } & \multicolumn{4}{|c|}{ Full-field ERG } \\
\hline & \multicolumn{2}{|c|}{ Photopic } & \multicolumn{2}{|c|}{ Scotopic } \\
\hline & $O D$ & OS & $O D$ & OS \\
\hline 1 & $\mathrm{~N}$ & $\mathrm{~N}$ & $\mathrm{~N}$ & $\mathrm{~N}$ \\
\hline 2 & $\mathrm{~N}$ & $\mathrm{~N}$ & RA, NIT & $\mathrm{N}$ \\
\hline 3 & NR & NR & RA, IIT & RA, IIT \\
\hline 4 & NA, IIT & NA, IIT & RA, IIT & RA, IIT \\
\hline 5 & RA, IIT & RA, IIT & RA, IIT & RA, IIT \\
\hline 6 & $\mathrm{~N}$ & $\mathrm{~N}$ & $\mathrm{~N}$ & $\mathrm{~N}$ \\
\hline 7 & $\mathrm{~N}$ & $\mathrm{~N}$ & $\mathrm{~N}$ & $\mathrm{~N}$ \\
\hline
\end{tabular}

ERG results are expressed in abbreviations (different setups were used in different centres): ERG=electroretinography; IIT=increased implicit time; $\mathrm{N}=$ normal amplitude and implicit time; $\mathrm{NIT}=$ normal implicit time; $\mathrm{NR}=$ not recorded; $\mathrm{OD}=$ oculus dexter; $\mathrm{OS}=$ oculus sinister; $\mathrm{RA}=$ reduced amplitude.

Heidelberg, Germany), the optical and technical principles of which have been described in detail previously. ${ }^{4,30,31}$ An argon blue laser (HRA classic) or an optically pumped solid state laser (HRA II) are used for excitation at $488 \mathrm{~nm}$. A barrier filter allowed to restrict detection of emitted light to a wavelength range above $500 \mathrm{~nm}$. Digital images are processed using a flexible frame processor and subsequently displayed on a computer screen. FAF images were recorded in accordance with a standard operation procedure, including focussing in the red-free reflection mode $(\lambda=514 \mathrm{~nm}$ for HRA classic, $\lambda=488 \mathrm{~nm}$ for HRA II), acquisition of a series of images $(488 \mathrm{~nm})$ and calculation of mean images after automated alignment (out of nine single images) in order to amplify signal to noise ratio using image analysis software (Heidelberg Eye Explorer, Heidelberg Engineering, Heidelberg, Germany). ${ }^{11}$

FAF images of normal eyes show a typical decrease in FAF intensity in the macular area due to absorption by macular pigment in the neurosensory retina anterior to the LF-containing RPE cell monolayer. ${ }^{32-35}$ There is also a lower signal along large retinal vessels (absorption) and at the optic disc (absence of autofluorescent material).

\section{Fundus-controlled microperimetry}

Fundus-controlled microperimetry was performed with dilated pupils with the Microperimeter 1 (MP1; Nidek Technologies, Padova, Italy), which allows determination of fundus-controlled light increment sensitivity (LIS) of photopic function. During examination, an integrated infrared fundus camera allows real-time fundus imaging on a monitor. In addition to predefined testing grids, individual testing points were chosen by the examiner on the fundus image, which is taken before the perimetric examination. An integrated eye tracking system continuously monitors the patients' eye movements and ensures exact spatial correlation between the anatomic landmarks and the perimetric sensitivity maps. Goldmann III stimuli were used. The stimuli were projected on a white background with background illumination set to 4 apostilbs (asb; $1.27 \mathrm{~cd} / \mathrm{m}^{2}$ ) and a stimulus presentation time of $100 \mathrm{~ms}$. Stimulus intensity could be varied on a $1 \mathrm{~dB}(0.1 \log )$-step scale from 0 to $20 \mathrm{~dB}$ (attenuation scale), where $0 \mathrm{db}$ represented the brightest luminance of $400 \mathrm{asb}\left(127 \mathrm{~cd} / \mathrm{m}^{2}\right){ }^{36-38}$

A reduction of the LIS was categorised as an 'absolute scotoma' when one or more stimuli were not seen with the brightest stimulus $(0 \mathrm{~dB}$, equivalent to an increased stimulus intensity of $20 \mathrm{~dB}$ ). It was categorized as a 'relative scotoma' when a circumscribed area of reduced LIS could be found. ${ }^{39}$

\section{Data analysis}

To compare the perimetric sensitivity map directly with FAF features, the former was superimposed on the FAF image using Adobe Photoshop Elements 3.0. software (Adobe Systems, Mountain View, CA, USA). A transparent FAF image allowed projecting the testing points from the MP1 image. To ensure that testing points were on the same location in MP 1 and FAF images, anatomic landmarks with high contrast, such as blood vessels, were used for superimposition.

\section{Statement of ethics}

We certify that all applicable institutional and governmental regulations concerning the ethical use of human volunteers were followed during this research.

\section{Results}

\section{Case history of patient 1 with PPRCA}

A 10-year-old Caucasian male patient was referred because of a positive family in his mother for RP. She was diagnosed in her early twenties in presence of typical fundus changes, nyctalopia, progressive concentric visual field loss, and abnormal ERG findings. The patient's father's ocular history was remarkable for difficulties in colour perception. The reported patient was the only child. Ophthalmic examination of the mother's and father's siblings as well as their children did not reveal any retinal abnormalities. Family history was unremarkable as was the patient's past medical history. At initial presentation the patient denied any visual symptoms.

On examination, visual acuity was 20/20. Goldmann perimetry revealed bilaterally scotomata arcing superior 
and inferior to fixation. No alterations on fundus examination were recorded at the time.

During a review period of 17 years the patient remained asymptomatic, and a visual acuity of 20/20 oculi uterque (OU) was sustained. He denied nyctalopia or photophobia. Full-field ERG at age 14 years showed normal scotopic and photopic responses. Funduscopic examinations revealed a normal calibre of the retinal vessels and a normal appearing optic nerve head. Chorioretinal atrophy as well as pigmentary changes with pigment migration into the neurosensory retina most pronounced along the temporal vascular arcades, incorporating the peripapillary area and extending towards the nasal arcades was noted in OU. Visual fields showed bilaterally scotomata arcing superior and inferior to fixation. On the basis of these findings the patient was diagnosed with PPRCA.

At age 21 mfERG findings objectively confirmed normal central retinal function with peak amplitudes within normal limits, while in the more eccentric elements, amplitudes were markedly reduced. FAF imaging revealed bilaterally an area of absent FAF along the arcades corresponding to the ophithalmoscopically visible atrophy and lines of increased FAF with crescentlike distribution surrounding the area of RPE atrophy (Figure 1a).
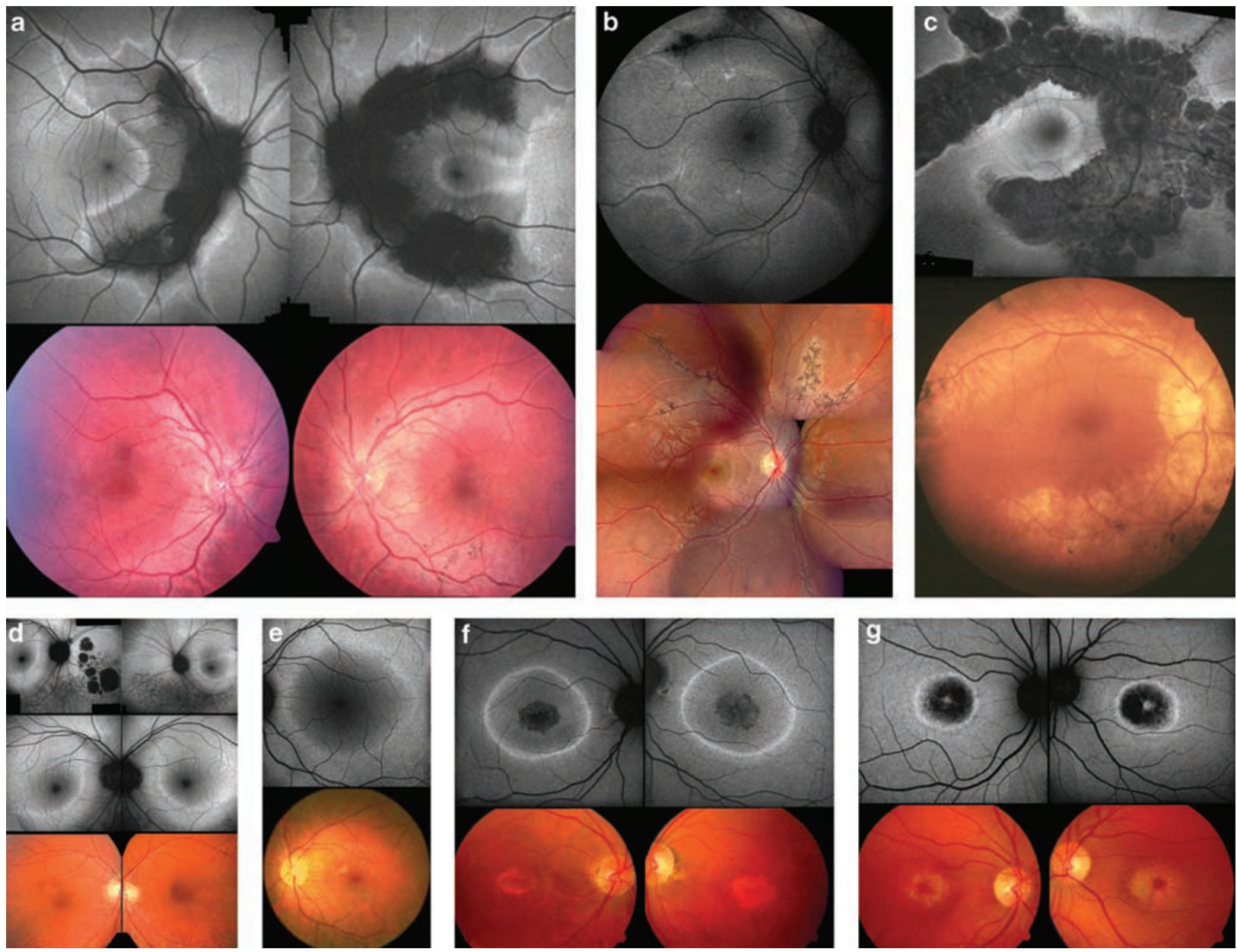

Figure 1 Arcs and rings of increased fundus autofluorescence (FAF) in patients with various forms of retinal dystrophies. Patients 1-3 $(\mathrm{a}-\mathrm{c})$ diagnosed with pigmented paravenous retinochoroidal atrophy (PPRCA) present an arc of increased FAF orientating along the retinal veins. There is a normal FAF signal between this arc and the atrophic areas (ie decreased FAF) and in the area that is not framed by the line. In the left eye of patient 1 (a) and the right eye of patient 3 (c), the arc of increased FAF almost merges to a parafoveal ring with a temporal opening. In patient 4 (d) with sector retinitis pigmentosa (RP), there is an arc with semi-circle structure in the parafoveal region. In typical RP (patient 5, e), there is a ring of increased FAF. Within and outside the ring, there is a normal FAF signal. In patient 6 (f) there is a parafoveal ring of increased FAF. Centrally, there is reduced FAF corresponding to the funduscopically visible lesion. On both sides of the ring, there is a normal FAF signal. In patient $7 \mathrm{~g}$ ) with bull's eye macular dystrophy, a ring of increased FAF borders directly the central lesion. In the very centre, there is a spot of preserved FAF. Note that there is no significant correlation of the arc of increased FAF in the conventional fundus photography. 


\section{PPRCA}

FAF imaging in PPRCA revealed an absent FAF signal along the larger retinal veins corresponding to funduscopically visible atrophy (Figure 1a-c). In variable distance to these atrophic areas, there was a well-defined arc of increased FAF. The contour of the arc had tiplike extensions towards the peripheral course of branching retinal veins (Figure 1a and $b$ ) and had a crescent-like distribution surrounding the area of RPE atrophy. In the left eye of patient 1 (Figure 1a) and the right eye of patient 3 (Figure 1c), the arcs almost merged to a parafoveal ring, while there was an opening towards the temporal side. The arc had no significant correlate on biomicroscopy. A retinal zone displaying a normal FAF signal was present between the area of absent FAF, ie atrophy, and the arc of increased FAF. A normal FAF signal was detected in the area peripheral to the arc of increased FAF.

Microperimetric assessment in patients 1 (Figure 2a) and 2 (Figure $2 b$ ) disclosed an absolute scotoma in the area of funduscopically visible chorioretinal atrophy. Severely reduced LIS was also noted peripheral to the atrophy in areas with normal appearing retina and normal FAF intensity. Superimposing the sensitivity map of the microperimetric examination on the FAF image revealed that the area of reduced LIS was sharply demarcated by the arc of increased FAF and not by the margin of absent FAF.

\section{Other retinal dystrophies}

In patient 4 (Figure 1d) with sector RP, there were patches of absent FAF nasally to the optic nerve head in the right eye. In both eyes, inferior to the optic nerve, there was an area of irregularly reduced FAF intensity. The fovea was surrounded by a semi-circle of high density within which FAF was preserved. The arc of increased FAF had no prominent finding on biomicroscopy. The area between the arc and the sectors of reduced FAF as well as uninvolved retinal sectors showed an apparently normal FAF signal.

In patient 5 (Figure 1e) with typical RP, there was a ring of increased FAF in the parafoveal region that had no prominent correlate on biomicroscopy. Inside and outside this ring, there was a normal FAF signal. In the retinal periphery, biomicroscopy revealed typical bone spicule hyperpigmentations. FAF was irregularly reduced in these areas.

In patient 6 (Figure 1f) with macular dystrophy, there was a central area of decreased FAF bilaterally corresponding to the funduscopically visible lesion. Peripheral to this lesion there was a ring of increased FAF that could not be associated with any prominent findings on biomicroscopy. On both sides of the ring there was a normal FAF signal.

In patient 7 (Figure 1g), a ring of increased FAF almost directly bordered the central lesion. Outside the ring, there was a normal FAF signal.

Microperimetry in typical RP (patient 5) revealed reduced LIS outside the ring of increased FAF despite a normal FAF signal in the corresponding area. Within the ring of increased FAF, LIS was preserved (Figure 3a).

Microperimetric assessment in patient 6 with macular dystrophy showed severely reduced LIS in the entire area inside the ring of increased FAF, independent of a decreased or normal FAF signal (Figure 3b). Outside the ring, LIS was preserved. These findings were opposite to the findings in patient 5 with RP where LIS was preserved inside and reduced outside the ring (Figure 3a).
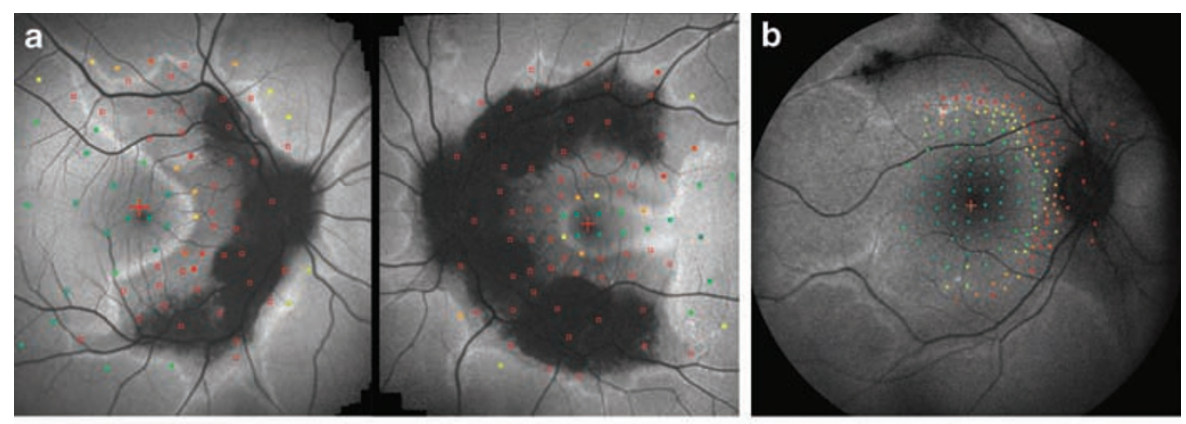

Attenuation scale $(\mathrm{dB})$

$\begin{array}{llllllllllll}2 & 4 & 6 & 8 & 10 & 12 & 14 & 16 & 18 & 20\end{array}$

Figure 2 Fundus-controlled microperimetric assessment in patient 1(a) with bilateral and patient 2(b) with unilateral pigmented paravenous retinochoroidal atrophy (PPRCA). The sensitivity map is superimposed on the fundus autofluorescence (FAF) image. Light increment sensitivity (LIS) varies from 0 to $20 \mathrm{~dB}$ (attenuation scale). Hollow red squares indicate testing points where the brightest stimulus was not seen. In the area that is framed by the arc of increased FAF, LIS is severely impaired independently of a normal or abnormal FAF signal. The arc of increased FAF demarcated the area of preserved LIS. 

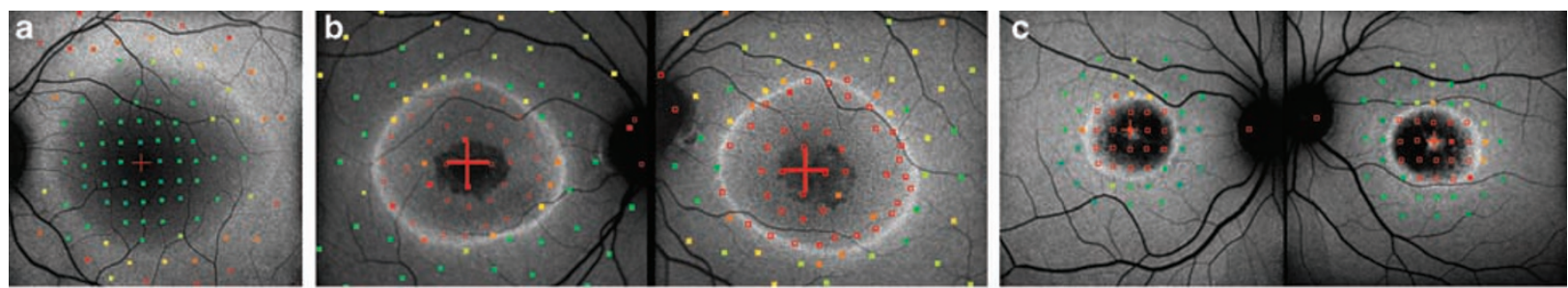

Attenuation scale $(\mathrm{dB})$

$\begin{array}{llllllllll}2 & 4 & 6 & 8 & 10 & 12 & 14 & 16 & 18 & 20\end{array}$

Figure 3 Fundus-controlled microperimetric assessment in respect of rings of increased fundus autofluorescence (FAF). The sensitivity map is superimposed on the FAF image. Light increment sensitivity (LIS) varies from 0 to $20 \mathrm{~dB}$ (attenuation scale). Hollow red squares indicate testing points where the brightest stimulus was not seen. In the patient with retinitis pigmentosa (RP) (patient 5 , a), LIS is preserved within the ring; outside, there is severely impaired LIS despite of a normal FAF signal. In patient 6 with macular dystrophy (b), LIS is significantly impaired within the ring independently of a normal or decreased FAF signal; outside, LIS is preserved. These findings are inverted to the finding in patient 5 (a) diagnosed with RP. In patient 7 with macular dystrophy (c), LIS is preserved outside the ring. In the very centre of the atrophic lesion, there is a patch of preserved FAF that correlates with less severe deterioration of LIS.

In patient 7, LIS was severely impaired in the atrophic area; in the very centre, less severe deterioration of LIS corresponded to a spot of preserved FAF (Figure 3c). LIS was within normal limits outside the ring of increased FAF.

\section{Serial FAF imaging}

Serial FAF imaging in patient 1 with PPRCA revealed marked extension of the area of decreased FAF - corresponding to the extension of the atrophic area visible on biomicroscopy - over a time period of 6 years. The arc of increased FAF showed only minimal migration in some areas (Figure 4a).

In patient 6 with macular dystrophy, there was slight spread of the area of decreased FAF, corresponding to the extent of the central atrophic lesion noted on biomicroscopy, and extension of the diameter of the ring of increased FAF within a time period of 10 years (Figure 4b).

\section{Discussion}

This study indicates that arcs of increased FAF in PPRCA and other retinal dystrophies that have no obvious correlation on fundus biomicroscopy are of functional significance. The orientation of such arcs may vary in different retinal dystrophies and may evolve differently with time. However, the similar appearance on FAF images and the concordance of functional findings indicate that these arcs in heterogeneous diseases share a common underlying pathophysiological mechanism.

In patients with $\mathrm{RP}^{14-19} \mathrm{LCA},{ }^{19,20}$ bull's eye maculopathy, ${ }^{21}$ XLRS, ${ }^{19,22}$ best macular dystrophy, ${ }^{23}$ cone dystrophy, and cone-rod dystrophy, ${ }^{19,24-28}$ an arc of increased FAF in form of a ring has been described. An arc of increased FAF eccentric to atrophic areas has been described in a single case report in PPRCA. ${ }^{40} \mathrm{FAF}$ findings in a patient with bilateral sector RP have recently been reported but no line of increased FAF has been observed. ${ }^{41}$

In this study, this ring structure was reproduced in RP and macular dystrophy, and a semi-circle structure was detectable in sector RP and advanced PPRCA, and tiplike extensions of such arcs towards branching retinal veins were present in PPRCA. It is remarkable, that the orientation of the arc of increased FAF along the retinal veins in eyes with more advanced PPRCA and in sector $\mathrm{RP}$ finally appears to result in the formation of a semicircle structure in the parafoveal region. This finding may indicate a related pathomechanism leading to formation and constriction of the parafoveal ring in RP that have been described previously. ${ }^{16}$ There was only minimal migration of the arc of increased FAF while there was marked progression of atrophy. This could also suggest that with time atrophy might 'overgrow' the arc and that it will subsequently disappear.

Functional analyses in respect of the ring of increased FAF have been performed in $\mathrm{RP}^{13,14,16-19}$ cone- and conerod dystrophy, XLRS, and LCA, ${ }^{19,28}$ by full-field and pattern ERG (PERG), fine matrix mapping, and multifocal ERG, respectively. In RP, microperimetric assessment has been performed. ${ }^{18}$ It has been concluded that the ring of increased FAF in RP demarcates areas of preserved central photopic function and that constriction of the ring may mirror progressive visual field loss by advancing photoreceptor dysfunction that encroaches over areas of central macular. ${ }^{14,16,17,19}$

It has been demonstrated that the PERG 50 amplitude - a valuable indicator of macular function - and the size of the abnormal ring show a high positive correlation in $\mathrm{RP}$, while in cone dystrophy and 

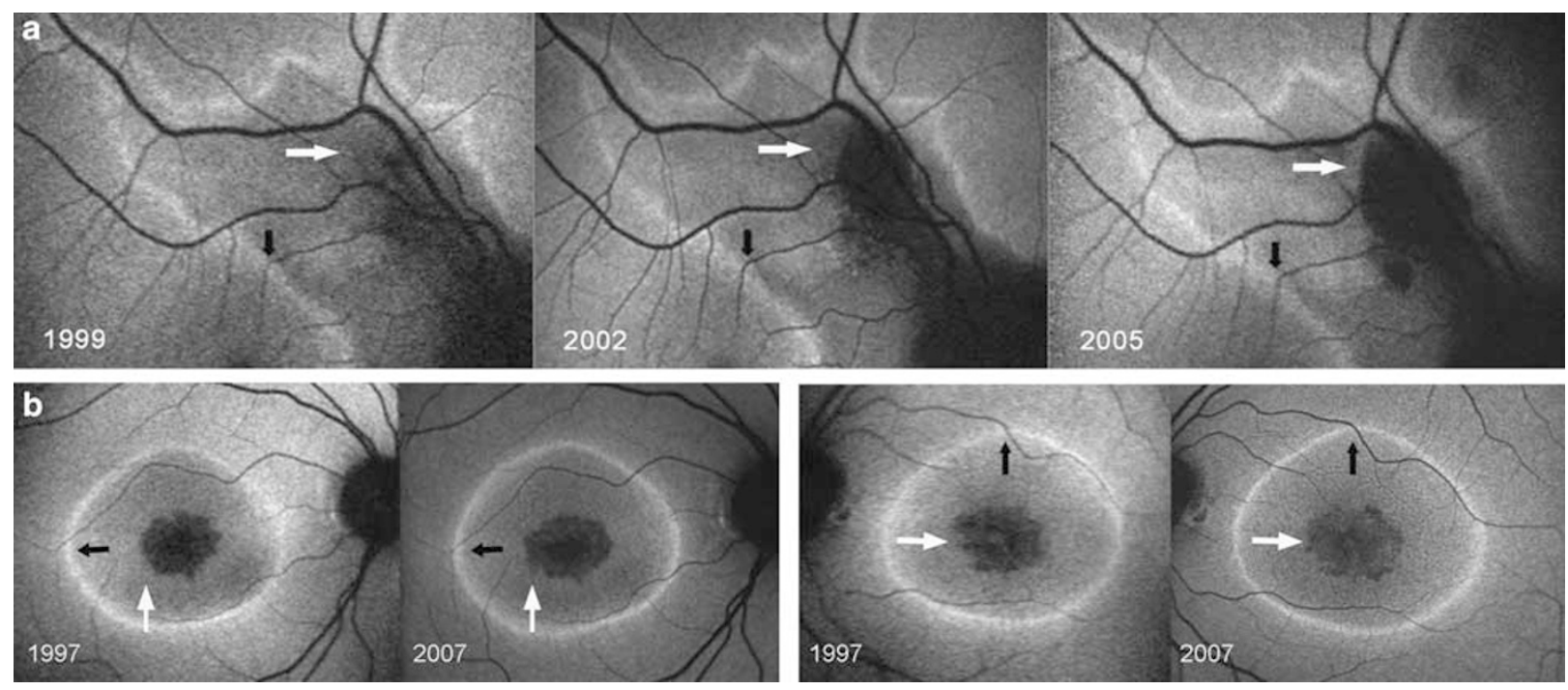

Figure 4 Serial fundus autofluorescence (FAF) imaging in pigmented paravenous retinochoroidal atrophy (PPRCA) and macular dystrophy. (a) Serial examination in patient 1 with PPRCA in the years 1999, 2002, and 2005 revealed marked extension of the area of decreased FAF (a, white arrow) that corresponded to a funduscopically noted extent of the atrophic area, and minimal migration of the arc of increased FAF (a, black arrow). (b) In patient 6 with macular dystrophy, FAF imaging in the years 1997 and 2007 (right eye on the left, left eye on the right side) revealed a slight spread of the area of decreased FAF (b, white arrow) - corresponding to a funduscopically visible extension of the central atrophic patch - and minimal but recognizable migration of the arc towards the periphery (b, black arrow).

cone-rod dystrophy, the PERG P 50 amplitude is inversely related to the size of the FAF ring. ${ }^{19,28}$

Our microperimetric results demonstrate that the actual situation is reciprocal in macular dystrophy compared to RP: in the two patients with macular dystrophy LIS was preserved outside the ring, while in RP, LIS was preserved centrally. Furthermore, as opposed to the finding that the ring constricts with time in $\mathrm{RP}^{16}$ we could detect an extension of the ring diameter in a patient with macular dystrophy within 10 years.

Enlargement of the ring has recently also reported in two patients with cone-rod dystrophy over a period of 20 months. $^{28}$

Increased FAF, and, therefore, excessive lipofuscin accumulation in RPE cells may result from an abnormally high turnover of photoreceptor outer segments or impaired RPE lysosomal degradation of normal or altered phagocytosed molecular substrates. ${ }^{2,42-45}$ We speculate that the arc of increased FAF reflects compensating RPE in an area where functional and dysfunctional photoreceptors are adjacent to each other. At this interface, there may be a higher metabolic load of the corresponding RPE cells with subsequent excessive accumulation of fluorophores in the lysosomal compartment.

It is remarkable that the FAF signal on both sides of this demarcation line was normal or near normal although on one side, LIS was severely impaired. Recent reports in individual patients with LCA and RP, respectively, indicated that there may also be severe retinal sensitivity loss despite preserved FAF. ${ }^{14,18-20}$ Robson et al. suggested that restoration of normal FAF intensity over concentric areas outside the ring in RP may indicate continued but possibly impaired phagocytosis and removal of abnormal material, or could be explained by loss of photoreceptor cells. ${ }^{14,16,17,19}$ Scholl et al ${ }^{20}$ concluded that a normal FAF signal reflects the presence of structurally intact photoreceptors and the integrity of the photoreceptor/RPE complex rather than normal photoreceptor function; it has been speculated that in those areas photoreceptor function could be rescued. ${ }^{19,20}$ These considerations demonstrate the value of FAF findings in specific retinal and macular dystrophies when therapeutic interventions will be attempted.

Since only photopic testing by microperimetry was performed here, investigations of the rod system function, for example FMM across the macula may offer further insight with respect to functional significance. FMM would also be superior in respect of the spatial resolution in relation to the narrowness of arcs of FAF.

In summary, an arc of increased FAF in different retinal dystrophies that may migrate with time appears to represent a demarcation of preserved retinal light sensitivity, independently of its orientation and localisation of functional impairment.

Arcs of increased FAF seem to represent a non-specific finding in different disease entities and points towards a common downstream pathogenetic pathway. FAF 
imaging in conjunction with fundus perimetry represents a non-invasive tool for 'functional mapping' in retinal dystrophies and may be considered as a secondary outcome measure in future therapeutic trials aiming at slowing progression of disease and additional loss of visual function in macular and diffuse retinal dystrophies associated with arcs of increased FAF signals.

\section{Acknowledgements}

This work was supported by DFG Heisenberg Fellowship SCHO 734/2-1; EU FP6, Integrated Project 'EVI-GENORET' (LSHG-CT-2005-512036), DFG Ho 1926/1-3, Ho 1926 3-1, and DOG research grant 2006.

\section{References}

1 Eldred GE, Katz ML. Fluorophores of the human retinal pigment epithelium: separation and spectral characterization. Exp Eye Res 1988; 47(1): 71-86.

2 von Rückmann A, Fitzke FW, Bird AC. Distribution of fundus autofluorescence with a scanning laser ophthalmoscope. Br J Ophthalmol 1995; 79(5): 407-412.

3 Delori FC, Dorey CK, Staurenghi G, Arend O, Goger DG, Weiter JJ. In vivo fluorescence of the ocular fundus exhibits retinal pigment epithelium lipofuscin characteristics. Invest Ophthalmol Vis Sci 1995; 36(3): 718-729.

4 Holz FG, Bellmann C, Margaritidis M, Schutt F, Otto TP, Volcker HE. Patterns of increased in vivo fundus autofluorescence in the junctional zone of geographic atrophy of the retinal pigment epithelium associated with age-related macular degeneration. Graefes Arch Clin Exp Ophthalmol 1999; 237(2): 145-152.

5 Spaide RF. Fundus autofluorescence and age-related macular degeneration. Ophthalmology 2003; 110(2): 392-399.

6 Sparrow JR, Boulton M. RPE lipofuscin and its role in retinal pathobiology. Exp Eye Res 2005; 80(5): 595-606.

7 Scholl HPN, Bellmann C, Dandekar SS, Bird AC, Fitzke FW. Photopic and scotopic fine matrix mapping of retinal areas of increased fundus autofluorescence in patients with agerelated maculopathy. Invest Ophthalmol Vis Sci 2004; 45(2): 574-583.

8 Schmitz-Valckenberg S, Bindewald-Wittich A, DolarSzczasny J, Dreyhaupt J, Wolf S, Scholl HP et al. Correlation between the area of increased autofluorescence surrounding geographic atrophy and disease progression in patients with AMD. Invest Ophthalmol Vis Sci 2006; 47(6): 2648-2654.

9 Holz FG, Bindewald-Wittich A, Fleckenstein M, Dreyhaupt J, Scholl HPN, Schmitz-Valckenberg S. Progression of geographic atrophy and impact of fundus autofluorescence patterns in age-related macular degeneration. $A m \mathrm{~J}$ Ophthalmol 2007; 143(3): 463-472.

10 Dandekar SS, Jenkins SA, Peto T, Scholl HPN, Sehmi KS, Fitzke FW et al. Autofluorescence imaging of choroidal neovascularization due to age-related macular degeneration. Arch Ophthalmol 2005; 123(11): 1507-1513.

11 Schmitz-Valckenberg S, Bultmann S, Dreyhaupt J, Bindewald A, Holz FG, Rohrschneider K. Fundus autofluorescence and fundus perimetry in the junctional zone of geographic atrophy in patients with age-related macular degeneration. Invest Ophthalmol Vis Sci 2004; 45(12): 4470-4476.

12 von Rückmann A, Fitzke FW, Bird AC. In vivo fundus autofluorescence in macular dystrophies. Arch Ophthalmol 1997; 115(5): 609-615.

13 Holder GE, Robson AG, Hogg CR, Kurz-Levin M, Lois N, Bird AC. Pattern ERG: clinical overview, and some observations on associated fundus autofluorescence imaging in inherited maculopathy. Doc Ophthalmol 2003; 106(1): 17-23.

14 Robson AG, El-Amir AN, Bailey CC, Egan CA, Fitzke FW, Webster AR et al. Pattern ERG correlates of abnormal fundus autofluorescence abnormalities in patients with retinitis pigmentosa and normal visual acuity. Invest Ophthalmol Vis Sci 2003; 44: 3544-3550.

15 Robson AG, Egan C, Holder GE, Bird AC, Fitzke FW. Comparing rod and cone function with fundus autofluorescence images in retinitis pigmentosa. In: La Vail MM, Hollyfield JG, Anderson RE (eds). Retinal Degenerations: Mechanisms and Experimental Therapy. Kluwer Academic/Plenum Publishers: New York, 2003, pp 41-47.

16 Robson AG, Saihan Z, Jenkins SA, Fitzke FW, Bird AC, Webster AR et al. Functional characterisation and serial imaging of abnormal fundus autofluorescence in patients with retinitis pigmentosa and normal visual acuity. $\mathrm{Br} J$ Ophthalmol 2006; 90(4): 472-479.

17 Robson AG, Egan CA, Luong VA, Bird AC, Holder GE, Fitzke FW. Comparison of fundus autofluorescence with photopic and scotopic fine-matrix mapping in patients with retinitis pigmentosa and normal visual acuity. Invest Ophthalmol Vis Sci 2004; 45(11): 4119-4125.

18 Popovic P, Jarc-Vidmar M, Hawlina M. Abnormal fundus autofluorescence in relation to retinal function in patients with retinitis pigmentosa. Graefes Arch Clin Exp Ophthalmol 2005; 243(10): 1018-1027.

19 Robson AG, Michaelides M, Saihan Z, Bird AC, Webster AR, Moore AT et al. Functional characteristics of patients with retinal dystrophy that manifest abnormal parafoveal annuli of high density fundus autofluorescence; a review and update. Doc Ophthalmol 2007; 116(2): 79-89.

20 Scholl HPN, Chong NHV, Robson AG, Holder GE, Moore AT, Bird AC. Fundus autofluorescence in patients with Leber congenital amaurosis. Invest Ophthalmol Vis Sci 2004; 45(8): 2747-2752.

21 Kurz-Levin MM, Halfyard AS, Bunce C, Bird AC, Holder GE. Clinical variations in assessment of bull's-eye maculopathy. Arch Ophthalmol 2002; 120(5): 567-575.

22 Tsang SH, Vaclavik V, Bird AC, Robson AG, Holder GE. Novel phenotypic and genotypic findings in X-linked retinoschisis. Arch Ophthalmol 2007; 125(2): 259-267.

23 Jarc-Vidmar M, Kraut A, Hawlina M. Fundus autofluorescence imaging in Best's vitelliform dystrophy. Klin Monatsbl Augenheilkd 2003; 220(12): 861-867.

24 Downes SM, Payne AM, Kelsell RE, Fitzke FW, Holder GE, Hunt DM et al. Autosomal dominant cone-rod dystrophy with mutations in the guanylate cyclase 2D gene encoding retinal guanylate cyclase-1. Arch Ophthalmol 2001; 119(11): 1667-1673.

25 Downes SM, Holder GE, Fitzke FW, Payne AM, Warren MJ, Bhattacharya SS et al. Autosomal dominant cone and conerod dystrophy with mutations in the guanylate cyclase activator 1A gene-encoding guanylate cyclase activating protein-1. Arch Ophthalmol 2001; 119(1): 96-105. 
26 Michaelides M, Holder GE, Webster AR, Hunt DM, Bird AC, Fitzke FW et al. A detailed phenotypic study of 'cone dystrophy with supernormal rod ERG'. Br J Ophthalmol 2005; 89(3): 332-339.

27 Ebenezer ND, Michaelides M, Jenkins SA, Audo I, Webster AR, Cheetham ME et al. Identification of novel RPGR ORF15 mutations in X-linked progressive cone-rod dystrophy (XLCORD) families. Invest Ophthalmol Vis Sci 2005; 46(6): 1891-1898.

28 Robson AG, Michaelides M, Luong V, Holder GE, Bird AC, Webster AR et al. Functional correlates of fundus autofluorescence abnormalities in patients with RPGR or RIMS1 mutations causing cone or cone-rod dystrophy. $\mathrm{Br} \mathrm{J}$ Ophthalmol 2007; 92(1): 95-102.

29 Marmor MF, Holder GE, Seeliger MW, Yamamoto S. Standard for clinical electroretinography (2004 update). Doc Ophthalmol 2004; 108(2): 107-114.

30 Jorzik JJ, Bindewald A, Dithmar S, Holz FG. Digital simultaneous fluorescein and indocyanine green angiography, autofluorescence, and red-free imaging with a solid-state laser-based confocal scanning laser ophthalmoscope. Retina 2005; 25(4): 405-416.

31 Bellmann C, Holz FG, Schapp O, Volcker HE, Otto TP. Topographie der Fundusautofluoreszenz mit einem neuen konfokalen scanning-laser-ophthalmoskop. Ophthalmologe 1997; 94(6): 385-391.

32 von Rückmann A, Fitzke FW, Bird AC. Fundus autofluorescence in age-related macular disease imaged with a laser scanning ophthalmoscope. Invest Ophthalmol Vis Sci 1997; 38(2): 478-486.

33 Snodderly DM, Brown PK, Delori FC, Auran JD. The macular pigment. I. Absorbance spectra, localization, and discrimination from other yellow pigments in primate retinas. Invest Ophthalmol Vis Sci 1984; 25: 660-673.

34 Robson AG, Moreland JD, Pauleikhoff D, Morrissey T, Holder GE, Fitzke FW et al. Macular pigment density and distribution: comparison of fundus autofluorescence with minimum motion photometry. Vision Res 2003; 43: 1765-1775.

35 Robson AG, Harding G, van Kuijk FJ, Pauleikhoff D, Holder GE, Bird AC et al. Comparison of fundus autofluorescence and minimum-motion measurements of macular pigment distribution profiles derived from identical retinal areas. Perception 2005; 34(8): 1029-1034.

36 Rohrschneider K, Springer C, Bultmann S, Volcker HE. Microperimetry - comparison between the micro perimeter 1 and scanning laser ophthalmoscope-fundus perimetry. Am J Ophthalmol 2005; 139(1): 125-134.

37 Springer C, Bultmann S, Volcker HE, Rohrschneider K. Fundus perimetry with the micro perimeter 1 in normal individuals: comparison with conventional threshold perimetry. Ophthalmology 2005; 112(5): 848-854.

38 Midena E, Radin PP, Convento E. Liquid crystal display microperimetry. Perimetry and the Fundus: An Introduction to Microperimetry, 1st edn. Slack Inc.: Thorofare, NJ, 2006, pp 15-26.

39 Charbel Issa P, Helb HM, Rohrschneider K, Holz FG, Scholl HPN. Microperimetric assessment of patients with Type 2 IMT. Invest Ophthalmol Vis Sci 2007; 48(8): 3788-3795.

40 Charbel Issa P, Scholl HP, Helb HM, Fleckenstein M, Inhetvin-Hutter C, Holz FG. [Unilateral Pigmented Paravenous Retinochoroidal Atrophy.]. Klin Monatsbl Augenheilkd 2007; 224(10): 791-793.

41 Meyerle CB, Fisher YL, Spaide RF. Autofluorescence and visual field loss in sector retinitis pigmentosa. Retina 2006; 26(2): 248-250.

42 Sanyal S, Hawkins RK. Development and degeneration of retina in rds mutant mice: altered disc shedding pattern in the albino heterozygotes and its relation to light exposure. Vision Res 1988; 28(11): 1171-1178.

43 Wells J, Wroblewski J, Keen J, Inglehearn C, Jubb C, Eckstein A et al. Mutations in the human retinal degeneration slow (RDS) gene can cause either retinitis pigmentosa or macular dystrophy. Nat Genet 1993; 3(3): 213-218.

44 Kennedy CJ, Rakoczy PE, Constable IJ. Lipofuscin of the retinal pigment epithelium: a review. Eye 1995; 9: 763-771.

45 Okubo A, Sameshima M, Unoki K, Uehara F, Bird AC. Ultrastructural changes associated with accumulation of inclusion bodies in rat retinal pigment epithelium. Invest Ophthalmol Vis Sci 2000; 41(13): 4305-4312. 